\title{
Ureteroiliac fistula secondary to radiotherapy in a patient with single renal metastasis of colon adenocarcinoma
}

\author{
Sarah Dormeus, MD; $;^{*}$ Erick A. Hernández, MD; Mickaël Nicolazzi, MD; Javier F. Barba, MD;" Rubén Algarra, \\ MD; Antonio Tienza, MD; 's Juan I. Pascual, MD, PhD; José M. Berián, MD, PhD; Juan J. Zudaire, MD, PhD
}

*Université Catholique de Louvain, Belgium; †Universidad Panamericana, Mexico; §Department of Urology, Clínica Universitad de Navarra, Spain

Cite as: Can Urol Assoc J 2013;7:E146-E1 49. http://dx.doi.org/10.5489/cuai.259

\section{Abstract}

We report the case of a 61-year-old man diagnosed in 2001 with rectal cancer (stage T3N1M0). The patient was treated with surgery, adjuvant chemotherapy and radiotherapy. In 2009, he was admitted to the urology department with a complaint of right hemiabdominal pain. The anatomopathological investigation reported renal metastasis of colon adenocarcinoma. After surgery, he received adjuvant chemotherapy. No tumour recurrence or metastasis was reported at the 22-month follow-up.

\section{Introduction}

The kidney is the fifth most frequent site of metastasis in tumours. However, secondary neoplasm only accounts for $3 \%$ of renal malignant disease. Ureteroiliac fistulas are infrequent but serious conditions, with a mortality rate that ranges from $7 \%$ to $23 \%$. We report these two rare conditions in a single patient.

\section{Case report}

A 61-year-old man was diagnosed in 2001 with moderately differentiated rectal cancer (stage T3N1M0). The patient was treated by surgery, adjuvant chemotherapy and radiotherapy. He was under medical control until September 2003, when the disease progressed to the level of colorectal anastomosis. This time, the right ureter was also involved with disease, the tumour was based on the right iliac vessels and infiltrated small intestine. Therapeutic approach consisted in surgery and radiotherapy in the tumoural area, followed by adjuvant chemotherapy.
In 2009, he was referred to the department of urology with complaints of right hemiabdominal pain for 2 days. $\mathrm{He}$ also presented with asthenia, dyspnea with minimal efforts and vomiting on two occasions before his admission to hospital.

Physical examination was only positive for right hemiabdominal tenderness and decreased bowel sounds. He was afebrile. Blood test showed anemia (hemoglobine levels: $7.4 \mathrm{~g} / \mathrm{dL}$ ) and hyperbilirrubinemia with cholestatic pattern. Creatinine levels were $1.7 \mathrm{mg} / \mathrm{dL}$. Abdominal x-ray did not revealed any abnormalities. One $20-\mathrm{cm}$ heterogeneous lesion in the right hemiabdomen, a grade IV/IV ureteral hydronephrosis in the right urinary tract and grade II/IV in the left side were seen on the abdominal ultrasound. These findings were correlated with the impaired renal function.

A computed tomography (CT) scan showed a small right pleural effusion, which confirmed bilateral ureteral hydronephrosis with a kidney size of $20 \times 17 \times 23 \mathrm{~cm}$. The radiologist report concluded a right ureteral hydronephrosis grade IV/IV, with large renal and urinary tract dilatation and compressive effect on the liver, the small intestine and the colon (Fig. 1, Fig. 2).

We catheterized the left urinary tract which resolved the hydronephrosis. After performing this procedure on the right side, we found blood circulating again. This sign was also observed when we tried to place a nephrostomy in the right kidney.

A renal arteriography revealed an irregular lesion in the middle zone of right common iliac artery. The finding was compatible with an ureteroiliac fistula, which was treated by stent-grafting of the right iliac artery. No complications occurred and the angiographic result was satisfactory.

Given the worsening of the anemia, the patient underwent a right radical nephrectomy. During the surgical procedure, we saw a lesion on the liver surface; we therefore performed a resection of second and third hepatic segments. 


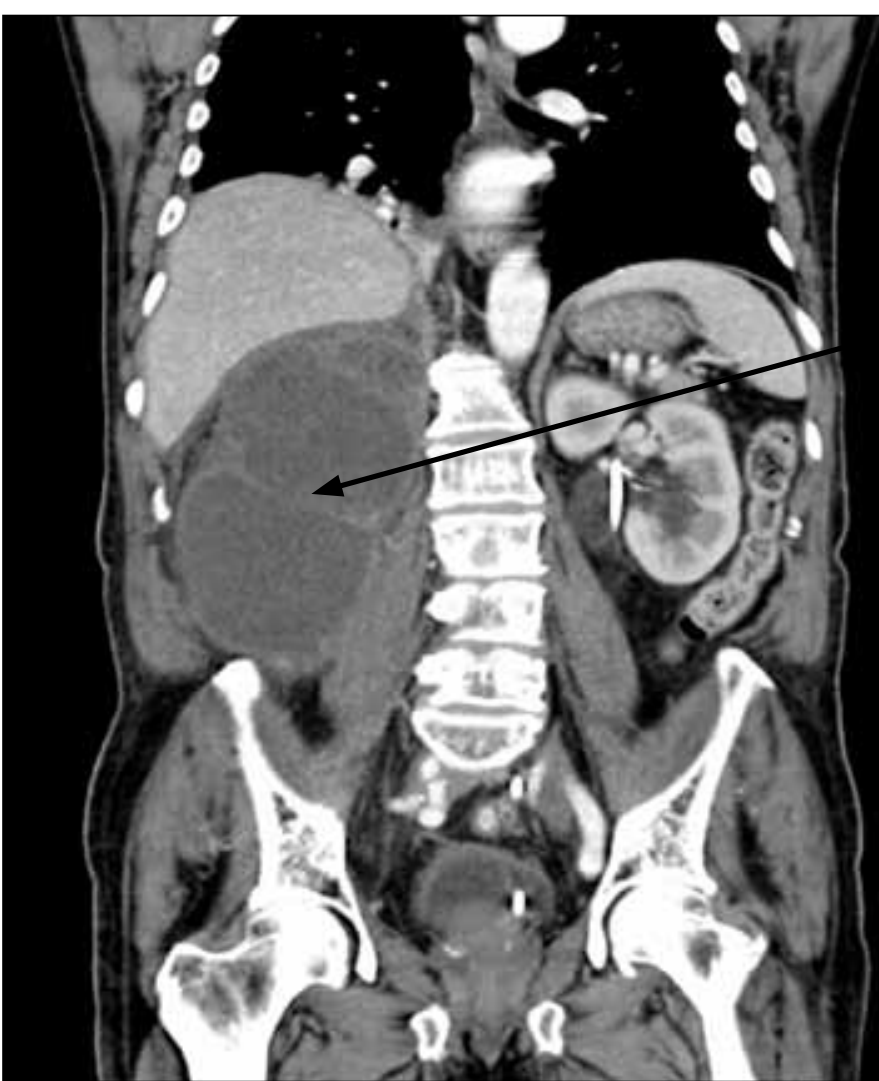

Fig. 1. A computed tomography scan showing right abdominal ureteral hydronephrosis (arrow) comprising liver and other adjacent structures.

The anatomopathological investigation reported right-sided renal metastasis of moderately differentiated colon adenocarcinoma and massively necrotic renal parenchyma.

Following the patient's uneventful recovery, the patient was discharged from hospital. Days later he resumed his treatment with adjuvant chemotherapy. No tumour recurrence or metastases were reported at the 22-month follow-up after completing his treatment. He had a normal renal function with a creatinine clearance of $1.2 \mathrm{mg} / \mathrm{dL}$ and a grade II/IV hydronephrosis requiring periodic stent replacements.

\section{Discussion}

The case we report deals with two situations: secondary renal tumours and uretero arterial fistulas. We will discuss these two rare conditions and focus on the second one.

\section{Secondary neoplasm of the kidney}

Tumours of the urinary tract (kidney and bladder) and male genital organs (prostate, testis and penis) are frequently reported in Europe and North America, although the genitourinary organs are not a common site for metastasis. Secondary renal neoplasms appear late in life, around the seventh decade, and are equally distributed among the sexes.
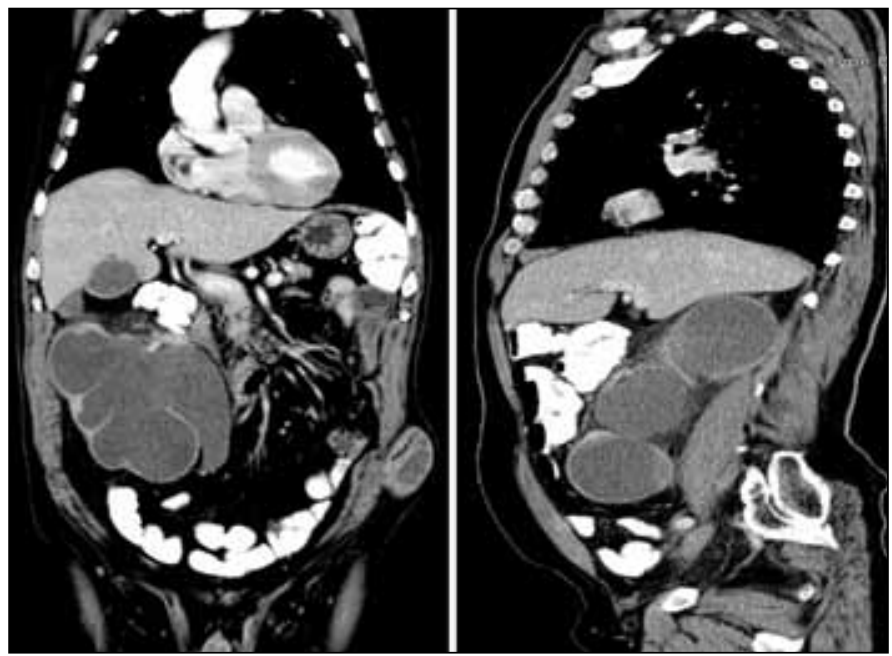

Fig. 2. A computed tomography scan showing right abdominal ureteral hydronephrosis comprising liver and other adjacent structures.

The final diagnosis of a secondary tumour is often hard to make. The collaboration between the urologist, pathologist and radiologist is the key in identifying the origin of a secondary neoplasm in the genitourinary system.

Bates and Baithun concluded that $1.6 \%$ to $3 \%$ of the solid malignant tumours surgically removed from the genitourinary tract are secondary neoplasms; $3 \%$ of renal solid malignancies are metastatic masses. ${ }^{1}$ Secondary neoplasms were found in $2.3 \%$ of the bladder tumour, $2.1 \%$ of the prostate tumour and $1.6 \%$ of the testis tumour. Of the subjects dying of malignant disease, renal metastasis were found in $9 \%$ to $20 \%$ of autopsies; in general, malignant disease is found in $1.8 \%$ to $7.2 \%$ of all autopsies.

The histological type of secondary kidney neoplasm is the adenocarcinoma (30\%), squamous-cell carcinoma (28\%), small-cell carcinoma (8\%) and malignant melanoma (6\%). The prognosis of adenocarcinoma (the most frequent type) is very poor because it occurs in patients affected by late-stage carcinomas. When the kidney is involved, the most common primary sources is lung $(44 \%)$, stomach $(9 \%)$, breast and colorectal (7\% each), oesophagus and pancreas ( $4 \%$ each) and uterus ( $2 \%)^{1}$

With a malignant disease spreads to the kidneys, bilateral and multiple lesions are generally observed. Metastasis as isolated renal mass is not common and little is known about its features and outcomes. ${ }^{2}$

Adamy and colleagues studied 13 patients who underwent nephrectomy for solitary metastasis to the kidney. ${ }^{3}$ They found that the most common primary site was the lung in 5 patients $(38 \%)$, followed by the colon in $2(15 \%)$, chest wall in $2(15 \%)$ and bone, brain, breast and salivary gland in 1 patient each (8\%). 


\section{Uretero arterial fistula}

The second issue of our clinical case is the ureteroarterial fistula caused by radiotherapy. These are uncommon but serious; the mortality rate ranges from $7 \%$ to $23 \% ;{ }^{4,5}$ when the diagnosis is not made before surgery, the mortality could reach $58 \% .{ }^{6}$ Rapid diagnosis followed by appropriate treatment significantly reduces the mortality.

\section{Risk factors}

Most ureteroarterial fistulas are seen in a predisposing context which leads to a degeneration of the vascular and ureteral walls. Several conditions can explain the formation of fistulas between an iliac artery and the ureter: underlying vascular pathology, previous surgery (genitourinary, pelvic and vascular operations), previous radiotherapy (although only in combination with surgery) and endoureteral procedures (e.g., prolonged use of ureteral stents or catheters, especially rigid ones, ureterolithotomy complicated with a urinary leakage). ${ }^{7-11}$ Like our patient, most people developing these fistulas have a history of pelvic malignancy. Infection and pregnancy are also risk factors. ${ }^{12}$

In most patients, fistulas are localized at the pelvic rim where the ureters cross the common iliac artery; the common iliac has been reported to be involved more often than the internal or external iliac arteries. ${ }^{12,13}$

\section{Symptoms}

Hematuria is the principal symptom of ureteroiliac fistula, from minor hematuria to massive hemorrhage leading to hypovolemic chock, but one of the most frequent presentation is an intermittent bleeding followed by a massive hematuria during the replacement of a ureteral stent. This wide range of symptoms may complicate the diagnosis. The patient may also complain of having flank pain probably secondary due to clot formation within the renal pelvis and ureter, causing distension of the urinary tract. Our patient only came with hemiabdominal pain, with no hematuria.

Early diagnosis is key to decreasing the risk of mortality. A high level of suspicion, without waiting for a massive hematuria to confirm the condition, will encourage an aggressive diagnostic approach during the preoperative period. Moderate or minor hematuria in a patient with risk factors should be followed appropriately.

\section{Diagnostic}

Unfortunately they are no certain methods to diagnose the fistula. Cystoscopy can help to localize the bleeding at one of the ureteral orifices. Retrograde pyelography may help to diagnose fistulas if there is a favourable ureteroarterial pres- sure gradient. Consequently, if there is significant bleeding, the sensitivity of pyelography decreases.

The sensitivity of the CT is too low to recommend it as the first-line exam; the changes associated with fistula may be subtle with no visible defects. If contrast is used, it may be possible to see the fistula.

The sensitivity of standard angiography has been demonstrated to be between $23 \%$ and $41 \%$ for detecting ureteroarterial fistulas ${ }^{8}$ (reaching $100 \%$ in a series ${ }^{[12]}$ ). Major extravasation of the contrast into the ureter is the most evident finding that may be seen in a secondary fistula. It is also possible to see slight irregularity of the arteria or small linear defects in the contrast which may be compare to an intimal flap. In some cases, the angiography will initially appear as normal. This is noticed when a ureteral stent, still in place, momentarily obstructs the hole inside the ureter. We may need a selective catheterization of the internal iliac artery to show subtle defects in the vessel which is affected by the fistula.

\section{Treatment}

Multiple therapeutic choices are available. ${ }^{7-9,14}$ They must resolve the arterial and ureteral lesions. The vascular defect can be repaired by an open approach: local reconstruction, ligature of the vessel with or without extra-anatomic reconstruction (this reconstruction includes a femoro-femoral crossover bypass).

Endovascular procedures, like embolization (with or without extra anatomic reconstruction) and graft-covered stent are attractive and popular therapeutic options. Knowing that the common or external iliac arteries are most frequently involved in ureteroarterial fistulas, the vascular surgeon should be consulted before choosing embolization. This endovascular approach will disrupt arterial support to the leg, which explains the necessity to perform a femoro-femoral bypass graft after embolization. On the other hand, stent-grafting allows excluding the fistula to the ureter and preserving arterial vascularization of the inferior limb. No graft infection (potentially caused by graft contamination by urinary bacteria) has been reported after stent-grafting. No recurrence of ureteroarterial fistulas have been reported after such treatment.

We can say that occlusion setting in the iliac artery stent ranges from $53 \%$ to $77 \%$ in the 4 -year patency rate. However, the long-term patency rates of those stents is not well-known. ${ }^{15}$ The longest follow-up reported in 1997 was 12 months. ${ }^{16}$

In 2005, Krambeck and colleagues published an algorithm of the therapeutic methods (Fig. 3). They proposed a nephrectomy with ureterectomy or ureter ligation when the renal function is normal (contralateral kidney has a normal function) because in most patients it is the definitive 


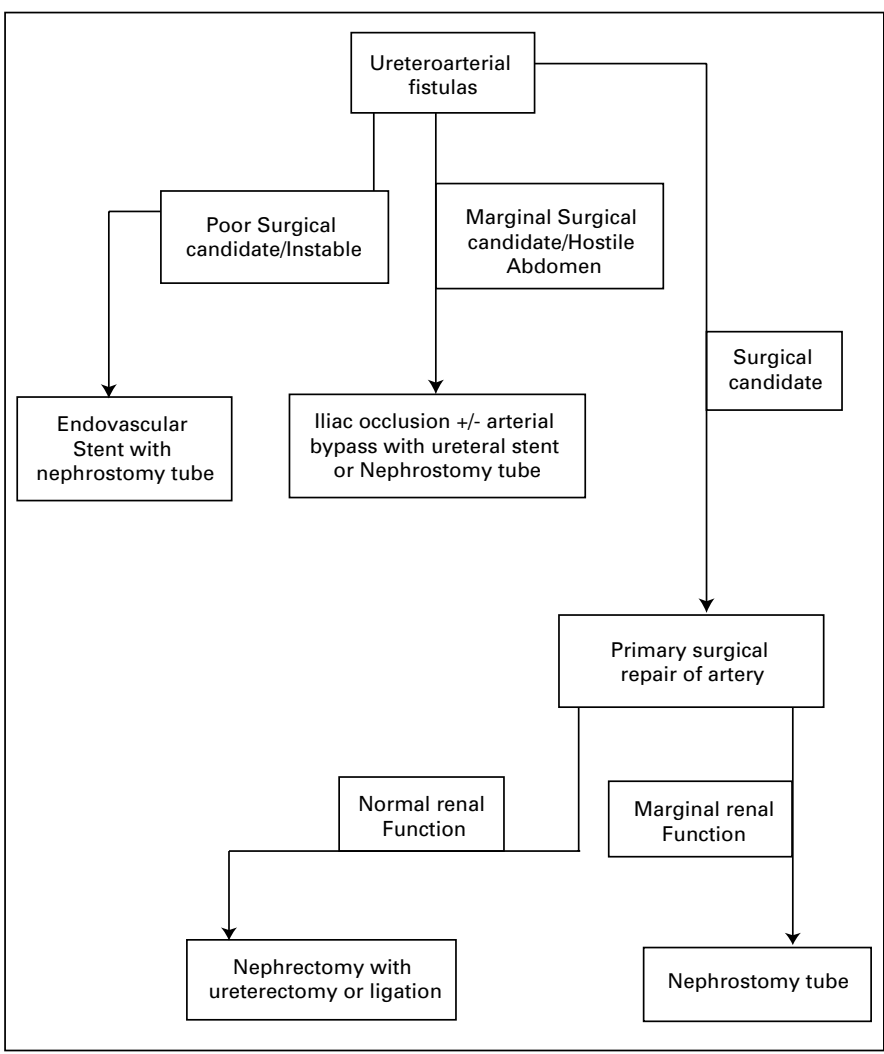

Fig. 3. Ureteroiliac artery fistula: treatment algorithm. Adapted from Krambeck et al. ${ }^{8}$

treatment for a life-threatening hematuria. Other types of reconstructive surgery are very difficult in radiated patients. Wound healing is often compromised, making major reconstruction less feasible.

\section{Conclusion}

Our case is extremely unusual; it gathers two rare conditions in a single patient. It is clear how early management for ureteroiliac fistulas is essential to decrease the rate of mortality. Even though arteriography is not completely accurate in diagnosing this condition, it was useful in identifying the lesion. We found that a history of surgery and radiotherapy near the zone of the fistula as risk factors. Our therapeutic approach was adequate, as stent-grafting is associated with the lesser recurrence rate after 12 months. However, our case shows that this therapy can be effective for a longer period of time.

Solitary isolated metastatic lesions are uncommon in kidney. For diagnostic purposes, knowing the medical history is crucial. This case also shows the relevance of teamwork between urology and other medical specialties.
Nephrectomy should not be considered a therapeutic option for all patients. Although it was justified in this case since both lesions involved other systems.

Competing interests: None declared.

This paper has been peer-reviewed.

\section{References}

1. Bates AW, Baithun SI. The significance of secondary neoplasms of the urinary and male genital tract. Virchows Arch 2002;440:640-7. http://dx.doi.org/10.1007/s00428-001-0549-x

2. Morichetti D, Mazzucchelli R, Lopez-Beltran A, et al. Secondary neoplasms of the urinary system and male genital organs. BJU Int 2009;104:770-6. http://dx.doi.org/10.1111/j.1464-410X.2009.08746.x.

3. Adamy A, Von Bodman C, Ghoneim T, et al. Solitary, isolated metastatic disease to the kidney: Memorial Sloan-Kettering Cancer Center experience. BJU Int 2011;108:338-42. http://dx.doi.org/10.1111/ j.1464-410X.2010.09771.x

4. Van den Bergh RC, Moll FL, de Vries JP, et al. Arterio-ureteral fistula: 11 new cases of a wolf in sheep's clothing. J Urol 2008;179:578-81. http://dx.doi.org/10.1016/i.juro.2007.09.087

5. Vandersteen DR, Saxon RR, Fuchs E, et al. Diagnosis and management of ureteroiliac artery fistula: value of provocative arteriography followed by common iliac artery embolization and extraanatomic arterial bypass graffing. J Urol 1997;158:754-8. http://dx.doi.org/10.1016/S0022-5347(01)64307-6

6. Kerns DB, Darcy MD, Baumann DS, et al. Autologous vein-covered stent for the endovascular management of an iliac artery-ureteral fistula: Case report and review of the literature. J Vasc Surg 1996;24:680-6. http://dx.doi.org/10.1016/S0741-5214(96)70084-8

7. Darcy M. Uretro-Arterial Fistulas Tech Vasc Interventional Rad 2009;12:216-21. http://dx.doi. org/10.1053/i.tvir.2009.09.005

8. Krambeck AE, Dimarco DS, Gettman MT, et al. Ureteroiliac artery fistula: diagnosis and treatment algorithm. Urology 2005;66:990-4. http://dx.doi.org/10.1016/j.urology.2005.05.036

9. Bergqvist D, Pärsson H, Sherif A. Arterio-ureteralfistula—a systematic review. Eur J Vasc Endovasc Surg 2001;22:191-6. http://dx.doi.org/10.1053/ejvs.2001.1432

10. Batter SJ, McGovern FJ, and Cambria RP. Ureteroarterial fistula: case report and review of the literature. Urology 1996;48:481-9. http://dx.doi.org/10.1016/S0090-4295(96)00202-6

11. Kim DH, Mahdy A, Mundra V, e tal. Ureteroarterial Fistula. Case Report Med 2009;326969.

12. Dervanian $P$, Castaigne D, Travagli JP, et al. Arterioureteral fistula after extended resection of pelvic tumours: report of three cases and review of the literature. Ann Vasc Surg 1992;6:362-9. http:// dx.doi.org/10.1007/BF02008794

13. Gelder MS, Alvarez RD, Partridge EE. Ureteroarterial fistulae in exenteration patients with indwelling ureteral stents. Gynecol Oncol 1993;50:365-70. http://dx.doi.org/10.1006/gyno.1993.1227

14. Bilbao Jl, Cosin 0, Bastarrika $G$, et al. Treatment of ureteroarterial fistulae with covered vascular endoprostheses and ureteral occlusion. Cardiovasc Intervent Radiol. 2005;28:159-63. http://dx.doi. org/10.1007/s00270-004-0196-0

15. Rodriguez HE, Eggener SE, Podbielski $\mathrm{FJ}$, et al. Occlusion of an intraluminal endovascular stent graft after treatment of a ureteral-iliac artery fistula. Urology 2002;60:912. http://dx.doi.org/10.1016/ S0090-4295(02)01902-7

16. Bosch JL, Hunink MG. Meta-analysis of the results of percutaneous transluminal angioplasty and stent placement for aortoiliac occlusive disease. Radiology 1997;204:87-96.

Correspondence: Dr. Javier F. Barba and Dr. Juan J. Zudaire, Departamento de Urología, Clínica Universidad de Navarra, Avda. Pío XII, 36. 31008 Pamplona, Spain; javiferbar@hotmail.com, jzudaire@unav.es 